\title{
Hipertiroidismo por doença de Graves durante a gestação
}

\author{
Hyperthyroidism due to Graves' disease during pregnancy
}

Carla Amaral de Almeida ${ }^{1}$, Leonardo Vieira Neto ${ }^{1}$, Sheila Mamede da Costa ${ }^{1}$, Alexandru Buescu², Mário Vaisman ${ }^{3}$

\section{RESUM0}

Objetivo: avaliar a influência do hipertiroidismo na evolução da gravidez e a necessidade de ajustes na dose de antitiroidianos neste período e no pós-parto. Métodos: avaliação prospectiva de dados clínicos e laboratoriais de treze gestações em onze mulheres com hipertiroidismo devido à doença de Graves, com dosagem de TSH e $\mathrm{T}_{4}$ livre a cada trimestre ou quatro semanas após ajuste do antitiroidiano. $\mathrm{O}$ objetivo foi manter o $\mathrm{T}_{4}$ livre no terço superior da normalidade usando a menor dose possível de antitiroidiano. Resultados: a média de idade no início da gravidez foi de 31,1 anos (23 a 41). Houve redução da dose de antitiroidiano em oito gestações $(69,5 \%)$ e, em duas, a droga foi suspensa. Antes da gravidez, a dose média de propiltiouracil era de $400 \mathrm{mg} / \mathrm{dia}$ (200 a 900) e a de metimazol, $45 \mathrm{mg} / \mathrm{dia}$ (20 a 60). Após o parto, a dose dos antitiroidianos foi reduzida para 200 e $30 \mathrm{mg} / \mathrm{dia}$, respectivamente. Uma paciente apresentou parto prematuro ( $36^{\text {a }}$ semana de gestação) e outra, recém-nato pequeno para a idade gestacional ( $2.000 \mathrm{~g}$ com 38 semanas de gestação). Houve um caso de natimortalidade. Não houve abortamentos ou anomalias congênitas. Após o parto, a dose de antitiroidiano foi aumentada em sete pacientes e mantida nas demais. Conclusões: recomendamos acompanhamento rigoroso de gestantes hipertiroidianas e titulação decrescente da dose dos antitiroidianos no decorrer da gestação, com o intuito de evitar o hipotiroidismo materno e suas conseqüências no desenvolvimento fetal. O acompanhamento após o parto dever ser cuidadoso, já que há a possibilidade de nova exacerbação do hipertiroidismo. O uso dos antitiroidianos foi seguro para as pacientes e sua prole.

PALAVRAS-CHAVE: Complicações na gravidez; Hipertiroidismo; Doença de Graves

\section{ABSTRACT}

Purpose: to evaluate the influence of hyperthyroidism during pregnancy and the necessity of changing antithyroid drug dose in this period and after delivery. Methods: prospective evaluation of clinical and laboratorial findings of thirteen pregnancies in eleven pregnant women with hyperthyroidism due to Graves' disease. These women were evaluated through TSH and serum free $\mathrm{T}_{4}$ at each trimester or four weeks after setting thionamide dosage. The goal was to maintain free $\mathrm{T}_{4}$ in the superior third of the normal range using the lowest possible thionamide dose. Results: the mean age at the beginning of the pregnancy was 31.1 years (23 to 41 ). The mean dosage of thionamide was reduced in eight pregnancies (69.5\%) and, in two, the drug was discontinued. Before pregnancy, mean propylthiouracil dose was $400 \mathrm{mg} /$ day (200-900) and mean methimazole dose was $45 \mathrm{mg} / \mathrm{day}$ (20-60). After delivery, antithyroid drug dose was 200 and $30 \mathrm{mg} / \mathrm{day}$, respectively. One patient presented premature labor (at 36 weeks) and another, a newborn small for gestational age ( $2.000 \mathrm{~g}$ at 38 weeks). There was one stillborn. There were no miscarriages or congenital anomalies. After labor, antithyroid drug dose was increased in seven patients and in the others the dose was maintained. Conclusions: we suggest close follow-up of pregnant women with hyperthyroidism and progressive reduction of thionamide dose during pregnancy to avoid maternal hypothyroidism and its consequences to fetal development. After labor, these women must be evaluated regarding their thyroid function because hyperthyroidism can worsen. Thionamide use is safe for the patients and their offspring.

KEYWORDS: Pregnancy complications; Hyperthyroidism; Graves' disease

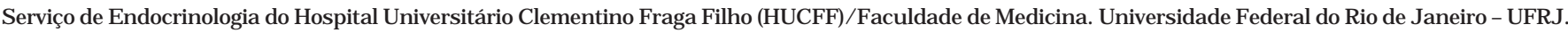

1 Pós-Graduandos do Serviço de Endocrinologia do HUCFF

2 Prof. Adjunto da Faculdade de Medicina da Universidade Federal do Rio de Janeiro - UFRJ - Rio de Janeiro (RJ) - Brasil

3 Professor Adjunto da Faculdade de Medicina da Universidade Federal do Rio de Janeiro - UFRJ, Chefe do Serviço de Endocrinologia do HUCFF Correspondência: Mário Vaisman

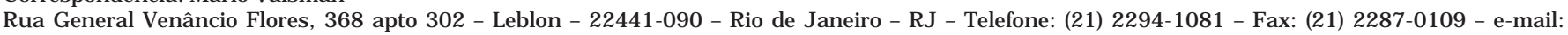
vaisman@hucff.ufrj.br

Recebido em: 18/2/2005

Aceito com modificações em: 11/4/2005
} 
Introdução

A gestação promove várias alterações hormonais e metabólicas no organismo materno, destacando-se os efeitos sobre a função tiroidiana. Além disso, disfunções tiroidianas auto-imunes diagnosticadas previamente à gravidez também podem apresentar alterações no seu curso ${ }^{1}$.

O hipertiroidismo durante a gravidez é pouco prevalente, sendo estimado em 1 a 2 casos em cada 1000 gestações ${ }^{1,2}$. Dentre as causas de hiperfunção tiroidiana na gravidez a mais freqüente é a doença de Graves. Porém, existem aquelas típicas da gestação, incluindo doença molar e tirotoxicose transitória da gravidez (também denominada tirotoxicose gestacional). Os níveis de tirotropina (TSH) podem estar suprimidos e, eventualmente, indetectáveis no primeiro trimestre (entre a $9^{\mathrm{a}}$ e a $13^{\mathrm{a}}$ semana) ${ }^{3}$ da gravidez, devido ao pico de gonadotrofina coriônica (hCG) observado neste periodo. Este fato pode ser observado em 10 a $20 \%$ das gestantes eutiroidianas ${ }^{1}$. Niveis muito elevados de hCG podem estar associados a hiperêmese gravídica.

Um conceito importante é o de que o hipertiroidismo está associado a uma série de complicações maternas e fetais, tais como préeclâmpsia, eclâmpsia, malformações fetais, parto prematuro e recém-nascido pequeno para a idade gestacional (PIG). Tem sido também relatada maior incidência de edema pulmonar, crise tirotóxica e arritmias cardiacas em gestantes hipertiroidianas $^{2}$. Quanto pior o controle da hiperfunção tiroidiana, maior é o risco para estas complicações ${ }^{1,4}$. Portanto, deve-se aconselhar a paciente com diagnóstico de hipertiroidismo e que deseja engravidar que obtenha controle da função tiroidiana antes da concepção, com a finalidade de reduzir a taxa de complicações. O tratamento do hipertiroidismo durante a gravidez ainda é objeto de discussões. Não há qualquer dúvida quanto á necessidade de compensar o quadro de tirotoxicose com a utilização de drogas antitiroidianas (AT), usando-se a menor dose possível que mantenha os níveis de $\mathrm{T}_{4}$ livre $\left(\mathrm{T}_{4} \mathrm{l}\right)$ próximos do limite superior da normalidade, afim de evitar o hipotiroidismo fetal. Posteriormente, caso necessário pela gravidade do quadro, ou se mantém a droga antitiroidiana ao longo de toda a gestação (casos leves a moderados) ou se encaminha a paciente para tratamento cirúrgico, no segundo trimestre (casos mais graves, refratários ao tratamento clínico). Como não é comum mulheres com hipertiroidismo descompensado engravidarem, é escassa a literatura sobre o tema, particularmente no âmbito nacional.
O objetivo deste estudo foi avaliar a influência do hipertiroidismo por doença de Graves na evolução da gravidez e a necessidade de ajustes na dose das drogas antitiroidianas neste período e no pós-parto.

\section{Métodos}

Foram acompanhadas, prospectivamente, 13 gestações em 11 pacientes com diagnóstico prévio de hipertiroidismo por doença de Graves durante o período de janeiro de 2001 a dezembro de 2004 . A média de idade ao engravidar foi de 31,1 anos (23 a 41). O diagnóstico de doença de Graves foi feito, em média, 18 meses ( 2 a 48) antes da gestação, devido à presença de hipertiroidismo associado a bócio difuso e/ou oftalmopatia leve. Em sete gestações, as pacientes já estavam em uso de propiltiouracil (PTU) e em quatro, de metimazol (MMI) quando foi diagnosticada a gravidez. Uma paciente que estava em uso de PTU e outra de MMI antes de engravidar suspenderam a medicação por iniciativa própria logo após o diagnóstico da gravidez, tendo sido orientadas a retornar a medicação na dose habitual (200 e $40 \mathrm{mg} /$ dia, respectivamente). O seguimento foi iniciado logo após a confirmação da gravidez, estando todas as pacientes no primeiro trimestre. Uma paciente apresentou rash cutâneo no início da gestação, tendo sido optado pela troca de PTU por MMI, não havendo intercorrências. Todas foram submetidas à dosagem de TSH e $\mathrm{T}_{4} 1$ (fração livre da tetraiodotironina) na época do diagnóstico da gravidez e a cada trimestre, pelo menos. O objetivo era manter o $\mathrm{T}_{4} 1$ no terço superior da normalidade.

$\mathrm{O}$ TSH e $\mathrm{T}_{4} 1$ foram dosados pelo método de quimioluminescência (kit DPC Immulite 2000), sendo os valores de referência, respectivamente, de 0,4 a 4,0 mU/L e de 0,8 a 1,9 ng/dL.

Todas as pacientes foram submetidas a consultas de pré-natal, em instituições próximas às suas residências. O número de consultas variou entre 2 e 11 . As pacientes foram orientadas a levar cópia de cada exame solicitado para as consultas em nosso serviço.

Após o parto, todas as pacientes continuaram em seguimento no ambulatório de Endocrinologia do Hospital Universitário Clementino Fraga Filho.

\section{Resultados}

O grau de tirotoxicose melhorou em todas as pacientes, conforme evidenciado pelo alívio dos sinais e sintomas de hipertiroidismo e pela re- 
dução do níveis de $\mathrm{T}_{4} 1$. A dose média dos AT foi reduzida em oito casos $(69,5 \%)$, sendo que em dois a droga foi suspensa. Antes da gravidez, a dose média de PTU era de 400 mg/dia (200 a 900) e a de MMI era de $45 \mathrm{mg} /$ dia (20 a 60) e no final da gestação de aproximadamente $200 \mathrm{mg} /$ dia (0 a 400) e $30 \mathrm{mg} /$ dia (0 a 60), respectivamente. A média dos niveis de $\mathrm{T}_{4} 1$ antes da gestação era de $2,6 \mathrm{ng} / \mathrm{dL}$ $(1,0$ a 4,3) e no final da gestação, de 1,4 ng/dL $(0,9$ a 1,8$)$. Nenhuma paciente chegou ao final da gravidez com niveis anormais de $\mathrm{T}_{4} 1$.
Não ocorreram complicações maternas. Uma paciente apresentou parto prematuro (idade gestacional de 36 semanas) e outra, recém-nato PIG (2.000 g com idade gestacional de 38 semanas). Houve um natimorto. A média de peso ao nascer destas crianças foi de $2.967 \mathrm{~g}$ (2.000 a 3.800). Não houve abortamentos ou ocorrência de malformações congênitas. Os resultados obtidos estão descritos na Tabela 1.

Tabela 1 - Características das grávidas com doença de Graves, droga empregada e desfecho da gravidez.

\begin{tabular}{ccccccccc}
\hline Pacientes & $\begin{array}{c}\text { Idade } \\
\text { (anos) }\end{array}$ & Droga & $\begin{array}{c}\text { Dose pré } \\
(\mathbf{m g})\end{array}$ & $\begin{array}{c}\text { Dose final } \\
(\mathbf{m g})\end{array}$ & $\begin{array}{c}\mathrm{T}_{4} \text { l início } \\
(\mathbf{n g} / \mathrm{dL})\end{array}$ & $\begin{array}{c}\mathrm{T}_{4} \text { I final } \\
(\mathrm{ng} / \mathrm{dL})\end{array}$ & $\begin{array}{c}\text { Parto } \\
\text { Peso ao nascer } \\
\text { (gramas) }\end{array}$ \\
\hline 1 & 27 & PTU & 300 & 200 & 2,1 & 1,5 & /AT & 2.560 \\
2 & 26 & PTU & 200 & 400 & 1,8 & 1,3 & Natimorto & 2.000 \\
3 & 23 & PTU & 400 & 100 & 1,0 & 0,9 & AT & 3.480 \\
4 & 34 & PTU & 300 & 400 & 3,7 & 1,5 & AT & 3.260 \\
5 & 32 & MMI & 60 & 60 & 2,0 & 1,6 & AT & 2.000 \\
6 & 31 & MMI & $0 / 40$ & 40 & 1,9 & 1,6 & AT & 3.400 \\
7 & 29 & MMI & 20 & 0 & 1,4 & 0,9 & AT & 3.800 \\
8 & 35 & MMI & 40 & 15 & 3,9 & 1,8 & AT & 3.200 \\
9 & 30 & MMI & 50 & 20 & 4,3 & 0,9 & 36 semanas & 3.530 \\
10 & 36 & PTU/MMI & 600 & 40 & 3,8 & 1,4 & AT & 3.210 \\
11 & 34 & PTU & 100 & 200 & 2,9 & 1,6 & AT & 3.030 \\
12 & 41 & PTU & $0 / 200$ & 0 & 1,5 & 1,1 & AT & 2.450 \\
13 & 26 & PTU & 900 & 400 & 3,0 & 1,8 & AT & 2.650 \\
\hline
\end{tabular}

AT: a termo; PTU: propiltiouracil; MMI: metimazol.

No pós-parto, a dose de AT foi aumentada em sete casos e mantida em outros cinco. Não tivemos acesso aos dados do pós-parto em uma paciente. A dose média no pós-parto foi de $40 \mathrm{mg} /$ dia para o MMI e de $400 \mathrm{mg} /$ dia para o PTU. As pacientes foram orientadas a fazer uso da medicação logo após a amamentação.

Os recém-nascidos foram submetidos a testes de rastreamento para diagnóstico de disfunção tiroidiana (teste do pezinho), sendo todos negativos.

\section{Discussão}

Durante a gestação, o hipertiroidismo secundário a doenças auto-imunes tende a melhorar progressivamente a partir do segundo trimestre, já que no primeiro pode ocorrer exacerbação pela elevação dos níveis de $\mathrm{hCG}^{5}$. Estudo recente ${ }^{3}$ mostrou elevação significativa dos valores médios de TSH, sendo esta elevação progressiva após cada trimestre.

Algumas hipóteses foram sugeridas para explicar a melhora do hipertiroidismo e de outras doenças auto-imunes na gestação, tais como: ocorrência de imunossupressão parcial durante a gravidez, com redução dos títulos de anticorpos antitiroidianos maternos; aumento dos níveis da proteína ligadora de tiroxina (TBG) e conseqüente redução da fração livre; perda de iodo específica da gestação pelo aumento da depuração renal; e a hipótese de que o balanço entre anticorpos estimuladores e bloqueadores do receptor de TSH pode favorecer os últimos ${ }^{1}$. No pós-parto há nova tendência de exacerbação do hipertiroidismo ${ }^{2,5}$.

O tratamento de escolha do hipertiroidismo durante a gestação é o uso das drogas AT. O tratamento cirúrgico está indicado apenas quando não se consegue obter o eutiroidismo clínico e laboratorial ou quando a dose de AT necessária para se obter o eutiroidismo materno induz 
hipotiroidismo fetal (diagnosticado pelo atraso na idade óssea e bradicardia). Nestes casos, a tiroidectomia subtotal deve ser realizada preferencialmente no segundo trimestre, embora não tenha sido documentada maior incidência de abortamento caso a cirurgia seja realizada no primeiro trimestre ${ }^{2}$. Nenhuma das nossas pacientes necessitou de tratamento cirúrgico, o que está de acordo com dados da literatura, que relatam ser rara a indicação cirúrgica nestes casos ${ }^{2}$.

Nossos resultados revelaram que em oito das treze gestações $(69,5 \%)$ a dose dos AT foi reduzida. Dados da literatura demonstram que a necessidade do uso de AT é menor no decorrer da gestação, sendo que em um terço das pacientes é possível a suspensão da droga nas últimas semanas ${ }^{2}$. É aconselhável manter o uso de AT até a $32^{\text {a }}$ semana de gestação para prevenir recidiva ${ }^{2}$.

Com relação ao AT de escolha, ainda é sugerido o uso preferencial de PTU ${ }^{1,2,4}$. Esta decisão baseia-se no fato de existir menor transferência placentária deste quando comparado ao MMI. Além disso, não foram descritas anomalias congênitas com o uso de PTU. O uso de MMI não está contraindicado na gestação. Porém, alguns estudos revelaram aumento da incidência de aplasia cutis congênita (ausência de pele tipicamente em uma área de 0,5 a $3,0 \mathrm{~cm}$ na região occipital) ${ }^{6,7}$ e de outras embriopatias graves (atresia de coanas, fístulas esofágica-traqueal, unhas hipoplásicas, anormalidades faciais e atraso psicomotor $)^{8}$. Entretanto, nem todos os autores concordam com a necessidade de troca do MMI por PTU durante a gestação $^{2,9}$. Das nossas pacientes, seis $(46,1 \%)$ foram tratadas com MMI e, embora tenha sido uma pequena amostra, não foi observado nenhum caso de malformação congênita.

O objetivo do tratamento na gestação é manter os niveis de $\mathrm{T}_{4} 1$ no limite superior da normalidade, utilizando-se a menor dose possivel de AT. É preferivel um aumento na dose da medicação do que manter níveis inaceitáveis de $\mathrm{T}_{4} 1^{4}$, já que $\mathrm{o}$ risco de ocorrerem complicações materno-fetais conseqüentes ao hipertiroidismo é maior quanto mais grave for a hiperfunção tiroidiana. Pode-se dividir as pacientes em três grupos: aquelas que se mantêm eutiroidianas desde o início da gestação, aquelas com hipertiroidismo no início da gravidez, mas que obtiveram controle da hiperfunção tiroidiana até seu término, e aquelas que se mantém hipertiroidianas durante toda a gravidez. Observa-se aumento da ocorrência de baixo peso ao nascer, prematuridade e eclâmpsia apenas no terceiro grupo de pacientes. Outros estudos demonstraram aumento da prevalência de recém-natos PIG, caso a paciente permanecesse hipertiroidiana por mais de 30 semanas durante a gestação ${ }^{10}$, além de maior chance de natimortalidade entre os recém-nascidos de pacientes sem tratamento adequado da hiperfunção tiroidiana ${ }^{11}$.

Contudo, ainda é incerto se o hipertiroidismo poderia estar associado à ocorrência de malformações fetais, com alguns estudos mostrando incidência semelhante à da população geral ${ }^{10} \mathrm{e}$ outros, aumento ${ }^{12,13}$. Nossos resultados não revelaram a ocorrência de malformações fetais, porém uma paciente apresentou parto prematuro e outra, recém-nascido PIG. Além disso, houve um caso de natimortalidade. Nesta última paciente foi necessário o aumento da dose de AT pois, apesar de o $\mathrm{T}_{4} 1$ no início da gestação ter sido considerado adequado, houve elevação dos níveis deste no decorrer da gestação, por provável uso irregular do AT. Dados semelhantes são vistos na população brasileira e do estado do Rio de Janeiro ${ }^{14}$.

Além destas possíveis complicações, existe também o risco de hipertiroidismo neonatal. Esta é condição pouco freqüente, ocorrendo em apenas 1 a $2 \%^{1,2}$ das gestações de pacientes com doença de Graves. Trata-se de condição relacionada à presença de anticorpos estimuladores maternos e que apresenta altas taxas de morbidade e mortalidade. A possibilidade de recorrência em uma futura gestação é pequena ${ }^{2}$.

Recomenda-se que as doses média e máxima de PTU sejam de $100 \mathrm{mg} / \mathrm{dia}^{2,4}$ e de $450^{4}$ a 600 $\mathrm{mg} / \mathrm{dia}^{2}$, respectivamente, ao passo que as de MMI sejam de $10 \mathrm{mg} / \mathrm{dia}^{2,4}$ e de $40^{4}$ a $60 \mathrm{mg} / \mathrm{dia}^{2}$. A dose média de AT em uso pelas nossas pacientes no final da gestação está dentro do limite recomendado, tendo sido de $200 \mathrm{mg} /$ dia para o PTU e de $30 \mathrm{mg} /$ dia para o MMI.

O tratamento excessivo da paciente pode levar à ocorrência de bócio e hipotiroidismo fetais. Niveis inapropriadamente baixos de hormônios tiroidianos para o feto podem ocasionar alterações no seu desenvolvimento, principalmente no que diz respeito ao sistema nervoso central ${ }^{1,15}$, e mesmo o hipotiroidismo materno leve pode ter impacto desfavorável na capacidade intelectual dos filhos destas pacientes ${ }^{16}$. Não há evidências de relação entre alterações no coeficiente de inteligência infantil e o uso de PTU ou MMI durante a gestação ${ }^{2,17,18}$. Entretanto, o hipotiroidismo fetal também pode ser conseqüente ao tratamento inadequado do hipertiroidismo durante a gestação. $\mathrm{O}$ hipertiroidismo materno causa ambiente de excesso de hormônios tiroidianos para o feto, o que prejudica o desenvolvimento do eixo hipotálamohipófise-tiróide, gerando hipotiroidismo central ${ }^{19}$. Os filhos de nossas pacientes vêm apresentando desenvolvimento neuropsicointelectual normal.

Observamos piora do grau de tirotoxicose pelo menos dois meses após o parto em sete ca- 
sos, o que obrigou a aumento da dose de AT. Esta necessidade de aumento na dose dos AT também está de acordo com dados da literatura, que demonstram ser possivel piora da tirotoxicose dois a 12 meses após o parto ${ }^{1,5}$.

Com os dados acima, concluímos que na gravidez pode-se observar mudanças favoráveis no curso de disfunções auto-imunes, como a doença de Graves. De fato, é possivel a redução e até mesmo a suspensão dos AT, devido à melhora progressiva do hipertiroidismo. A manutenção do eutiroidismo na gestante reduz o risco de complicações maternas e fetais observadas em pacientes com hipertiroidismo descontrolado. Porém, no pós-parto pode-se observar piora do grau de tirotoxicose, sendo necessário aumento da dose de AT. Assim, recomendamos acompanhamento rigoroso de gestantes hipertiroidianas, com dosagem de hormônios tiroidianos pelo menos uma vez por trimestre, além da titulação decrescente da dose dos AT no decorrer da gestação, com o intuito de evitar o hipotiroidismo materno e suas conseqüências no desenvolvimento fetal. Além disso, o acompanhamento após o parto também deve ser cuidadoso, já que há a possibilidade de nova exacerbação do hipertiroidismo.

\section{Referências}

1. Glinoer D. Management of hypo- and hyperthyroidism during pregnancy. Growth Horm IGF Res. 2003;13 Suppl A:S45-54

2. Mestman JH. Hyperthyroidism in pregnancy. Endocrinol Metab Clin North Am. 1998;27(1):127-49.

3. Sieiro Netto L, Coeli CM, Micmacher E, Mamede SC, Nazar LO, Correa ER, et al. Estudo longitudinal do eixo hipófise-tireóide durante a gravidez. Arq Bras Endocrinol Metab. 2004;48(4):493-8.

4. Mandel SJ, Cooper DS. The use of antithyroid drugs in pregnancy and lactation. J Clin Endocrinol Metab. 2001;86(6):2354-9.

5. Amino N, Tanizawa O, Mori H, Iwatani Y, Yamada $\mathrm{T}$, Kurachi K, et al. Aggravation of thyrotoxicosis in early pregnancy and after delivery in Graves' disease. J Clin Endocr Metab. 1982;55(1):108-12.

6. Mujtaba Q, Burrow GN. Treatment of hyperthyroidism in pregnancy with propylthiouracil and methimazole. Obstet Gynecol. 1975;46(3):282-6.
7. Van Dijke CP, Heydendael RJ, De Kleine MJ. Methimazole, carbimazole, and congenital skin defects. Ann Intern Med. 1987;106(1):60-1.

8. Johnsson E, Larsson G, Ljunggren M. Severe malformations in infant born to hyperthyroid women on methimazole. Lancet. 1997;350(9090):1520.

9. Millar LK, Wing DA, Leung AS, Koonings PP, Montoro MN, Mestman JH. Low birth weight and preeclampsia in pregnancies complicated by hyperthyroidism. Obstet Gynecol. 1994;84(6):946-9.

10. Mitsuda N, Tamaki H, Amino N, Hosono T, Miyai K, Tanizawa O. Risk factors for developmental disorders in infants born to women with Graves disease. Obstet Gynecol. 1992;80(3 Pt 1):359-64.

11.Davis LE, Lucas MJ, Hankins GD, Roark ML, Cunningham FG. Thyrotoxicosis complicating pregnancy. Am J Obstet Gynecol. 1989;160(1):63-70.

12. Momotani N, Ito K, Hamada N, Ban Y, Nishikawa Y, Mimura T. Maternal hyperthyroidism and congenital malformation in the offspring. Clin Endocrinol (Oxf). 1984;20(6):695-700.

13.Wing DA, Millar LK, Koonings PP, Montoro MN, Mestman JH. A comparison of propylthiouracil versus methimazole in the treatment of hyperthyroidism in pregnancy. Am J Obstet Gynecol. 1994;170(1 Pt 1):90-5.

14. Ministério da Saúde. Datasus. Informações de saúde. Estatísticas vitais: mortalidade e nascidos vivos. [citado 2005 jan 09]. Disponivel em www.datasus.gov.br/ tabnet/tabnet.htm\#EstatVitais

15. Gharib H, Cobin RH, Dickey RA. Subclinical hypothyroidism during pregnancy: position statement from the American Association of Clinical Endocrinologists. Endocr Pract. 1999;5(6):367-8.

16. Haddow JE, Palomaki GE, Allan WC, Williams JR, Knight GJ, Gagnon J, et al. Maternal thyroid deficiency during pregnancy and subsequent neuropsychological development of the child. N Engl J Med. 1999;341(8):549-55.

17. Eisenstein Z, Weiss M, Katz Y, Bank H. Intellectual capacity of subjects exposed to methimazole or propylthiouracil in utero. Eur $J$ Pediatr. 1992;151(8):558-9.

18. Messer PM, Hauffa BP, Olbricht T, Benker G, Kotulla $\mathrm{P}$, Reinwein D. Antithyroid drug treatment of Graves' disease in pregnancy: long-term effects on somatic growth, intellectual development and thyroid function of the offspring. Acta Endocrinol. 1990;123(3):311-6.

19. Kempers MJ, van Tijn DA, van Trotsenburg AS, de Vijlder JJ, Wiedijk BM, Vulsma T. Central congenital hypothyroidism due to gestacional hyperthyroidism: detection where prevention failed. J Clin Endocrinol Metab. 2003;88(12):5851-7. 